\title{
Crystal structure of cyclophilin A complexed with substrate Ala-Pro suggests a solvent-assisted mechanism of cis-trans isomerization
}

(x-ray crystallography/immunophilin/cyclosporin A/peptidyl-prolyl isomerase)

\author{
Hengming Ke*, Dale Mayrose, and Wei Cao \\ Department of Biochemistry and Biophysics, School of Medicine, The University of North Carolina, Chapel Hill, NC 27599
}

Communicated by William N. Lipscomb, December 17, 1992

\begin{abstract}
Cyclophilin is a binding protein for the immunosuppressive drug cyclosporin $A$ and is also an enzyme with peptidyl-prolyl cis-trans isomerase activity. The crystal structure of cyclophilin A complexed with the substrate AlaPro has been determined and refined to an $R$ factor of 0.196 at 1.64- $\AA$ resolution. The structure shows that only the cis form of Ala-Pro binds cyclophilin A despite the fact that Ala-Pro has an equilibrium majority of the trans form in solution. Simulation of the cis-trans isomerization in an ESV10 graphics system suggests a solvent-assisted mechanism in which first the peptidyl-prolyl bond is desolvated at the ground state by binding to the hydrophobic pocket of the active site, and later the intermediate state is stabilized by a hydrogen bond between the carbonyl oxygen of the amide bond and a bound water molecule.
\end{abstract}

Isomerization of a peptidyl-prolyl amide bond is a common reaction occurring in two structurally unrelated immunophilins: cyclophilin (CyP; refs. 1-3) and FK506-binding protein (4). It is also a slow step in protein unfolding or refolding (5). Because of its importance in the immunosuppression of T-cell activation and in protein folding, the cis-trans isomerization of the peptidyl-prolyl bond has received a great deal of attention in the past few years.

Several mechanisms have been proposed for the cis-trans isomerization: ( $a$ ) a covalent tetrahedral intermediate formed on the carbonyl carbon of the amide bond $(2,6),(b)$ a distortion mechanism in which an intermediate state with a twisted $\mathrm{C}-\mathrm{N}$ bond is stabilized by interactions with the enzyme (7), (c) a tetrahedral intermediate formed on the nitrogen atom of the amide bond (8), $(d)$ a catalysis by desolvation of the twisted amide intermediate by $\operatorname{CyP}(9,10)$, and $(e)$ a solvent-assisted mechanism that combines both the rotation of the amide bond and the impact of the $\mathrm{N}$ and $\mathrm{C}$ termini of the substrate on the rotation (11).

Although three-dimensional structures have been determined for the unligated recombinant human $\mathrm{T}$-cell cyclophilin A (CyPA) $(11,12)$ and for the human CyPA complexed with a tetrapeptide substrate $(13,14)$, insight into the cistrans isomerization has not been obtained, perhaps because of the poor binding of proline substrates and lack of highresolution structures. Here, we report the structure of the human T-cell CyPA complexed with the dipeptide Ala-Pro at 1.64- $\AA$ resolution. ${ }^{\dagger}$

\section{METHODS}

Crystals of the unligated human T-cell CyPA were grown at $4^{\circ} \mathrm{C}$ (12). The storage solution of $0.5 \mathrm{M}$ Ala-Pro was prepared by dissolving Ala-Pro in distilled water. The crystals of the unligated human CyPA were soaked in a solution containing

The publication costs of this article were defrayed in part by page charge payment. This article must therefore be hereby marked "advertisement" in accordance with 18 U.S.C. $\$ 1734$ solely to indicate this fact.
0.1 M Ala-Pro, $16 \mathrm{mM}$ Tris base, $5.4 \%$ (vol/vol) ethanol, and $32 \%(\mathrm{wt} / \mathrm{vol}$ ) polyethylene glycol (molecular weight, 3350$)$ at $\mathrm{pH} 8.5$ at room temperature for 9 days. The space group of the CyPA/Ala-Pro complex is $P 2_{1} 2_{1} 2_{1}$ with cell dimensions $a=$ $42.6 \AA, b=52.5 \AA$, and $c=89.3 \AA$. Diffraction data were collected at room temperature on a Rigaku phosphate image plate system in our laboratory. A total of 55,553 diffraction maxima were measured, resulting in 21,605 symmetryindependent reflections to a resolution of $1.64 \AA$ with an $R_{\text {merge }}$ of 0.051 . The data set was $89 \%$ complete to $1.64-\AA$ resolution, and $87 \%$ of the reflections had intensity stronger than $2 \sigma(I / \sigma)$.

The structure of the unligated human CyPA refined at 1.63- $\AA$ resolution (11) was used in the map calculation of the CyPA/Ala-Pro complex. The model was manually rebuilt by using the program FRODO linked to an ESV10 graphics system (15) and refined with the program XPLOR (16) on a Decstation 5000 computer. Molecular dynamics refinement (16) was applied to the CyPA/Ala-Pro structure without solvent molecules. At this stage, several rounds of model building and structural refinement brought the $R$ factor from 0.393 to 0.267 at $1.64-\AA$ resolution. After this refinement, solvent molecules were automatically picked up in the $F_{\text {obs }}-$ $F_{\text {calc }}$ map and incorporated into the coordinates. Only positions and $B$ factors were refined for the structure containing solvent molecules. After deletion of solvent molecules that had large $B$ factors in the coordinates, the next round of solvent pickup and structure refinement was repeated until no peaks stronger than $3.5 \sigma$ were found in the $F_{\text {obs }}-F_{\text {calc }}$ map.

\section{RESULTS AND DISCUSSION}

The final refinement against 21,073 reflections which were stronger than $2 \sigma$ at 6- to 1.64- $\AA$ resolution yielded an $R$ factor of 0.196 for the structure containing amino acid residues Asn ${ }^{2}$ to $\mathrm{Glu}^{165}$, the substrate Ala-Pro, and 80 water molecules. The root-mean-square deviations were $0.014 \AA$ and $2.6^{\circ}$ from the ideal geometries of bond length and bond angle, respectively. The average $B$ factors were $25.9 \AA^{2}$ for all atoms of CyPA and $25.5 \AA^{2}$ for the substrate Ala-Pro, indicating that the substrate Ala-Pro had full occupancy and a well-ordered conformation. $\mathrm{Asn}^{2}$ and $\mathrm{Glu}^{165}$, as well as a few side chains of the surface residues, had high $B$ factors $\left(50-80 \AA^{2}\right)$ and were partially disordered.

The structure of CyPA complexed with Ala-Pro (Fig. 1) is an eight antiparallel-stranded $\beta$-barrel, which is the same as the unligated human CyPA $(11,12)$. The binding of Ala-Pro does not change the molecular conformation of CyPA but does change the molecular orientation. When the CyPA/Ala-

Abbreviations: CyP, cyclophilin; CyPA, cyclophilin A; AAPA $N$-acetyl-Ala-Ala-Pro-Ala-amidomethylcoumarin; Wat ${ }^{n}$, water- $n$. *To whom reprint requests should be addressed.

†The atomic coordinates have been deposited in the Protein Data Bank, Chemistry Department, Brookhaven National Laboratory, Upton, NY 11973 (reference 1CYH). 


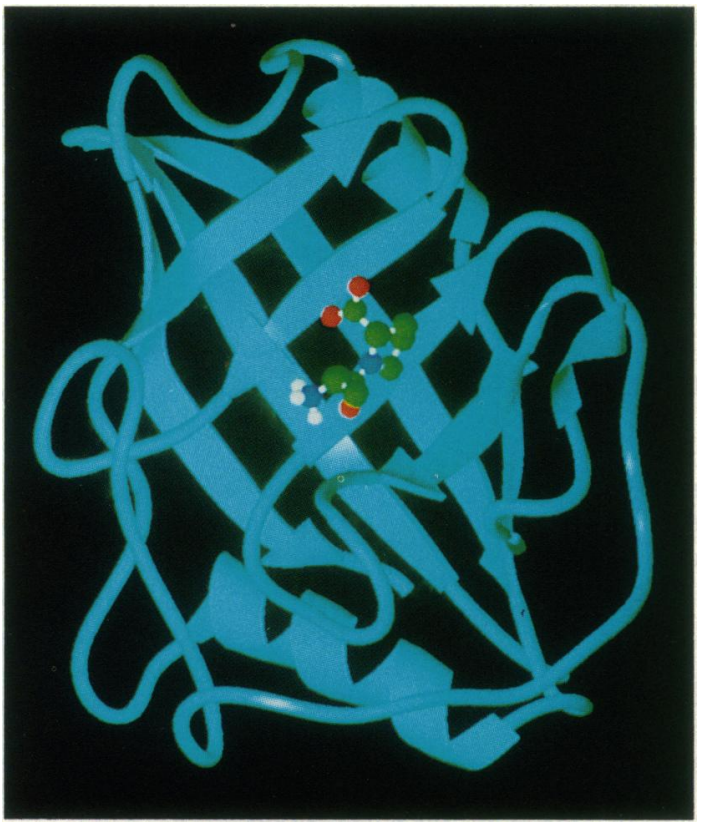

Fig. 1. Ribbon presentation of the CyPA/Ala-Pro structure. The substrate Ala-Pro binds to the surface of the eight-stranded $\beta$-barrel and is presented as a ball-and-stick model with oxygen red, carbon green, nitrogen dark blue, and hydrogen white.

Pro complex was superimposed on the unligated CyPA, a shifting of $\mathrm{C}^{\alpha}$ atoms with a root-mean-square difference of $0.39 \AA$ and a $2.5^{\circ}$ rotation of the molecular orientation was observed. The significant change of the molecular orientation is consistent with large differences between the diffraction data sets of the unligated CyPA and the CyPA/Ala-Pro complex. An $R$ factor of 0.398 was obtained by least-squares scaling of 12,233 structure factors common between the two data sets at 15 - to $2-\AA$ resolution. Nevertheless, careful inspection of the two structures revealed no substantial differences in the conformations of either the backbones or the side chains of CyPA due to the binding of Ala-Pro. Most active-site residues, such as $\mathrm{Arg}^{55}$ and $\mathrm{Phe}^{60}$, had the same conformations in both the unligated and Ala-Pro-complexed forms, but the backbones of Asn ${ }^{102}$ to Gly ${ }^{104}$ migrated slightly as shown by the root-mean-square error of $0.59 \AA$ in comparison to the overall difference of $0.39 \AA$ for the $\mathrm{C}^{\alpha}$ atoms.
One molecule of Ala-Pro binds each CyPA. There was no nonspecific binding of Ala-Pro to other sites due to soaking of the unligated crystals in the $0.1 \mathrm{M}$ Ala-Pro solution. Electron density in the $\left(2 F_{\text {obs }}-F_{\text {calc }}\right)$ map was well ordered and fitted a unique conformation of Ala-Pro (Fig. 2). The structure refined at $1.64-\AA$ resolution showed a conformational angle of $-3^{\circ}$ for the peptide bond, the cis form of the bound Ala-Pro, in contrast to $61 \%$ trans for free Ala-Pro in a normal solvent system (17).

The substrate Ala-Pro binds to the site, as predicted from the unligated structure of CyPA $(11,12)$. Residues which form either hydrophilic or hydrophobic interactions with Ala-Pro include $\mathrm{Arg}^{55}, \mathrm{Phe}^{60}, \mathrm{Met}^{61}, \mathrm{Gln}^{63}, \mathrm{Ala}^{101}, \mathrm{Asn}^{102}$, $\mathrm{Phe}^{113}$, Leu ${ }^{122}$, His ${ }^{126}$, and three water molecules (Table 1). The side chain of proline of the substrate is located in a hydrophobic pocket of CyP, which is composed of the side chains of Phe ${ }^{60}$, Met $^{61}$, Phe ${ }^{113}$, Leu ${ }^{122}$, and His ${ }^{126}$ (Fig. 3). The $\mathrm{N}$-terminal nitrogen of Ala-Pro forms three hydrogen bonds with the carbonyl oxygen of Asn ${ }^{102}$, water-206 (Wat ${ }^{206}$ ), and Wat $^{207}$, while the C-terminal carboxylic oxygen forms five with the side chains of $\mathrm{Arg}^{55}, \mathrm{Gln}^{63}$, Wat ${ }^{202}$, and Wat ${ }^{206}$ (Fig. 3 and Table 1 ). In addition, the carbonyl oxygen of the amide bond of the substrate is within a van der Waals radius of the nitrogen and carbonyl oxygen of $\mathrm{Asn}^{102}, \mathrm{C}^{\delta}$ of $\mathrm{His}^{126}$, and $\mathrm{C}^{\alpha}$ and $\mathrm{C}^{\beta}$ of $\mathrm{Ala}^{101}$ (Table 1).

There are eight bound water molecules at the active site. Five of them $\left(W^{200}{ }^{200}\right.$ Wat $^{201}$, Wat ${ }^{204}$, Wat ${ }^{206}$, and Wat ${ }^{207}$ ) are common to both the structure of the unligated CyPA and that of the CyPA/Ala-Pro complex (Table 2). Some of these water molecules may play a role in the cis-trans isomerization as discussed in the next section.

Role of His ${ }^{126}$. Although the importance of $\mathrm{His}^{126}$ in the cis-trans isomerization of the peptidyl-prolyl bond is obvious from the proximity of $\mathrm{His}^{126}$ to the substrate, its specific role in catalysis is uncertain. It was suggested that $\mathrm{His}^{126}$ was involved in attacking the prolyl amide bond on the basis of the structure of CyPA complexed with the tetrapeptide substrate $N$-acetyl-Ala-Ala-Pro-Ala-amidomethylcoumarin (AAPA) (13). However, further refinement of the same structure at 2.3- $\AA$ resolution showed neither hydrogen bonds nor indirect interactions via a water between $\mathrm{His}^{126}$ and the substrate (14), consistent with our structure of CyPA/Ala-Pro at $1.64-\AA$ resolution. Therefore, His ${ }^{126}$ is unlikely to directly attack the ground state of the substrate to induce transition of the amide bond.

In the structure of the CyPA/Ala-Pro complex, two imidazole nitrogen atoms of $\mathrm{His}^{126}, \mathrm{~N}^{\delta}$, and $\mathrm{N}^{\varepsilon}$, form two hydro-
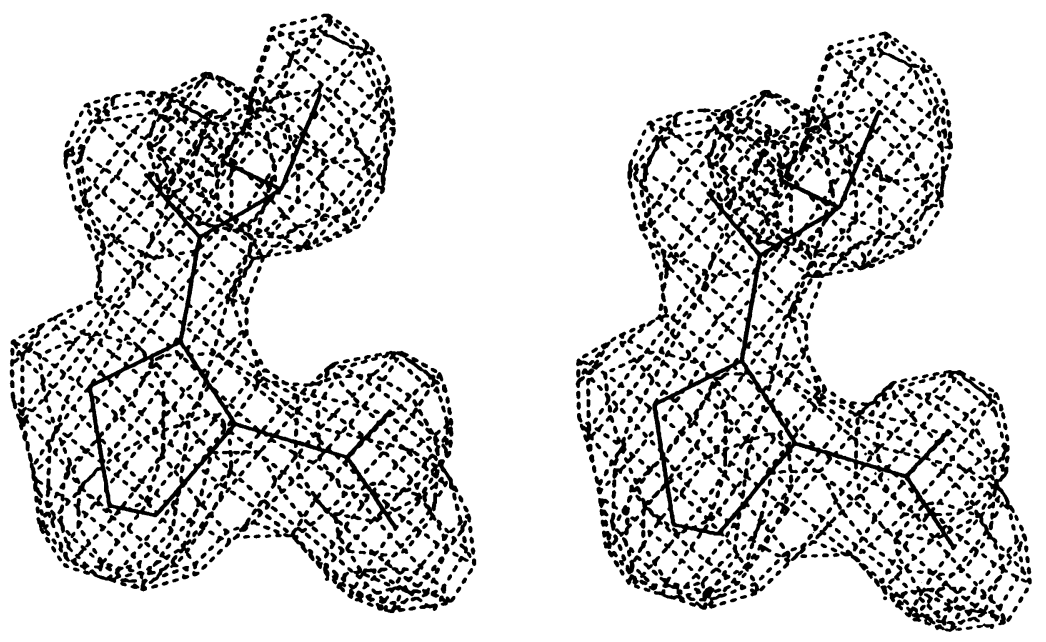

FIG. 2. Stereo picture of the bound form of Ala-Pro. The electron density (dotted lines) comes from the $2 F_{\text {obs }}-F_{\text {calc }}$ map and is contoured at $1.2 \sigma$. The substrate Ala-Pro has the highest density, $8 \sigma$, and an averaged $B$ value of $25.5 \AA^{2}$, comparable to the overall average $B$ value of $25.9 \AA^{2}$. Only the cis form of Ala-Pro with a peptide conformational angle of $-3^{\circ}$ binds to the enzyme. 
Table 1. Binding of Ala-Pro to CyPA

\begin{tabular}{|c|c|c|c|c|}
\hline $\begin{array}{c}\text { Substrate } \\
\text { atom }\end{array}$ & $\begin{array}{c}B \text { factor, } \\
\AA^{2}\end{array}$ & $\begin{array}{c}\text { Distance, } \\
\AA\end{array}$ & $\begin{array}{l}\text { CyPA } \\
\text { atom }\end{array}$ & $\begin{array}{l}\text { Bond } \\
\text { type* }\end{array}$ \\
\hline \multirow[t]{3}{*}{$\mathrm{Ala}^{1} \mathrm{~N}$} & 28.2 & 2.94 & $A_{s n}^{102} O$ & $\mathrm{H}$ \\
\hline & & 2.76 & Wat $^{206} \mathrm{O}$ & $\mathbf{H}$ \\
\hline & & 3.08 & $\mathrm{Wat}^{207} \mathrm{O}$ & $\mathrm{H}$ \\
\hline $\mathrm{Ala}^{1} \mathrm{CA}$ & 25.7 & 3.67 & $\mathrm{Ala}^{101} \mathrm{O}$ & V \\
\hline $\mathrm{Ala}^{1} \mathrm{CB}$ & 24.4 & 3.51 & $\mathrm{Asn}^{102} \mathrm{O}$ & V \\
\hline $\mathrm{Ala}^{1} \mathrm{C}$ & 26.2 & 3.85 & His $^{126} \mathrm{CE} 1$ & V \\
\hline \multirow[t]{5}{*}{$\mathrm{Ala}^{1} \mathrm{O}$} & 22.8 & 3.19 & $\mathrm{Asn}^{102} \mathrm{~N}$ & $\mathbf{P}$ \\
\hline & & 3.29 & Ala $^{101}$ CB & V \\
\hline & & 3.40 & $\mathrm{Ala}^{101} \mathrm{CA}$ & V \\
\hline & & 3.38 & $\mathrm{His}^{126} \mathrm{CE} 1$ & V \\
\hline & & 3.39 & $A s n^{102} \mathrm{O}$ & V \\
\hline Pro $^{2} \mathrm{~N}$ & 25.7 & 3.79 & Gln ${ }^{63}$ NE2 & V \\
\hline Pro $^{2} \mathrm{CA}$ & 24.9 & & None $^{\dagger}$ & \\
\hline Pro $^{2}$ CB & 24.2 & 3.82 & $\mathrm{Phe}^{60} \mathrm{CZ}$ & V \\
\hline Pro $^{2}$ CG & 23.9 & & None $^{\dagger}$ & \\
\hline \multirow[t]{4}{*}{ Pro $^{2} \mathrm{CD}$} & 23.2 & 3.62 & $\mathrm{Phe}^{113}$ CE1 & V \\
\hline & & 3.73 & Phe ${ }^{113}$ CD1 & V \\
\hline & & 3.71 & $\mathrm{His}^{126} \mathrm{CE} 1$ & V \\
\hline & & 3.89 & $\mathrm{His}^{126}$ NE2 & V \\
\hline \multirow[t]{3}{*}{ Pro $^{2} \mathrm{C}$} & 26.4 & 3.78 & Gln ${ }^{63}$ NE2 & V \\
\hline & & 3.55 & $\operatorname{Arg}^{55} \mathrm{NE}$ & V \\
\hline & & 3.78 & $\mathrm{Arg}^{55} \mathrm{NH} 1$ & V \\
\hline \multirow[t]{3}{*}{ Pro $^{2} \mathrm{O}$} & 27.0 & 2.92 & $\operatorname{Arg}^{55} \mathrm{NE}$ & $\mathbf{H}$ \\
\hline & & 2.89 & Gln ${ }^{63}$ NE2 & $\mathbf{H}$ \\
\hline & & 2.96 & $\mathrm{Wat}^{206} \mathrm{O}$ & H \\
\hline \multirow[t]{2}{*}{ Pro $^{2}$ OT } & 28.5 & 2.87 & $\mathrm{Arg}^{55} \mathrm{NH} 1$ & $\mathrm{H}$ \\
\hline & & 2.96 & $\mathrm{Wat}^{202} \mathrm{O}$ & $\mathbf{H}$ \\
\hline
\end{tabular}

Atom names are the same as defined in the program FRODO. Average $B$ factor over all atoms in CyPA is $25.9 \AA^{2}$.

${ }^{*} \mathrm{H}$, hydrogen; $\mathrm{V}$, van der Waals.

†No interactions are within $4-\AA ̊$ radius.

gen bonds with the carbonyl oxygen of $\operatorname{Met}^{100}(2.79 \AA)$ and Wat ${ }^{204}(2.88 \AA)$, respectively. These interactions also exist in the unligated CyPA structure (2.83 and $3.05 \AA$; ref. 11). The side chain of His ${ }^{126}$ is near the hydrophobic pocket of the active site but does not form hydrogen bonds with Ala-Pro. The closest distance is $3.38 \AA$, a van der Waals interaction between $\mathrm{C}^{\varepsilon}$ of $\mathrm{His}^{126}$ and the carbonyl oxygen of alanine of the substrate (Table 1 ). To verify the conformation of the side chain of His ${ }^{126}$, the imidazole plane of $\mathrm{His}^{126}$ was flipped by a $180^{\circ}$ rotation around the $\mathrm{C}^{\alpha}-\mathrm{C}^{\beta}$ bond in the ESV10
Table 2. Water molecules at the active site of CyPA/Ala-Pro

\begin{tabular}{|c|c|c|c|}
\hline Water & $\begin{array}{c}B \text { factor, } \\
\AA^{2}\end{array}$ & $\begin{array}{c}\text { Distance, } \\
\AA\end{array}$ & Atom \\
\hline \multirow[t]{4}{*}{$\mathrm{Wat}^{200}$} & 23.3 & 3.19 & $\operatorname{Trp}^{121} \mathrm{NE} 1$ \\
\hline & & 2.75 & Asn $^{106}$ OD1* $^{*}$ \\
\hline & & 2.92 & $\mathrm{Wat}^{202} \mathrm{O}$ \\
\hline & & 3.03 & Wat ${ }^{205} \mathrm{O}$ \\
\hline \multirow[t]{3}{*}{ Wat $^{201}$} & 61.2 & 2.72 & $\mathrm{His}^{54} \mathrm{NE} 1$ \\
\hline & & 3.19 & Gln ${ }^{63}$ OE2 \\
\hline & & 3.13 & $\mathrm{Wat}^{206} \mathrm{O}$ \\
\hline \multirow[t]{3}{*}{ Wat $^{202}$} & 29.1 & 2.96 & Pro $^{2}$ (substrate) OT \\
\hline & & 2.77 & $\mathrm{Wat}^{188} \mathrm{O}^{*}$ \\
\hline & & 3.02 & $\mathrm{Wat}^{203} \mathrm{O}$ \\
\hline Wat ${ }^{203}$ & 35.4 & 2.91 & $\operatorname{Asp}^{85} \mathrm{O}^{*}$ \\
\hline Wat 204 & 37.6 & 2.88 & $\mathrm{His}^{126}$ NE2 \\
\hline \multirow[t]{4}{*}{ Wat ${ }^{206}$} & 34.5 & 2.89 & Gln ${ }^{63}$ OE1 \\
\hline & & 2.76 & $\mathrm{Ala}^{1}$ (substrate) $\mathrm{N}$ \\
\hline & & 2.96 & Pro $^{2}$ (substrate) 0 \\
\hline & & 2.66 & Wat ${ }^{207} \mathrm{O}$ \\
\hline \multirow[t]{2}{*}{ Wat ${ }^{207}$} & 48.6 & 3.08 & $\mathrm{Ala}^{1}$ (substrate) $\mathrm{N}$ \\
\hline & & 2.98 & Gln 111 OE1 \\
\hline
\end{tabular}

$\mathrm{N}$ and $\mathrm{O}$ represent backbone nitrogen and carbonyl oxygen, respectively. Others are side-chain atoms.

*From symmetry-related molecule.

graphics system. This operation swapped the position of $C^{\delta}$ with $\mathrm{N}^{\delta}$ and that of $\mathrm{N}^{\varepsilon}$ with $\mathrm{C}^{\varepsilon}$. As a result, $\mathrm{N}^{\varepsilon}$ of $\mathrm{His}^{126}$ is the closest atom to the substrate Ala-Pro, but still $3.38 \AA$ away from the carbonyl oxygen of the amide bond. The flip of the imidazole plane of His ${ }^{126}$ did not create any new hydrogen bonds but broke two hydrogen bonds and placed two carbon atoms in unfavorable contacts with two oxygen atoms. Therefore, the conformation of $\mathrm{His}^{126}$ in our current structure is probably correct.

When the cis-trans isomerization was simulated on the ESV10 graphics system by manually rotating the amide bond, the carbonyl oxygen of the amide bond in the transition state of the substrate could be within hydrogen-bonding distance of $\mathrm{C}^{\varepsilon}$ of $\mathrm{His}^{126}$. Consequently, a possible mechanism may be composed of two steps: (i) the imidazole plane of $\mathrm{His}^{126}$ is flipped to place $\mathrm{N}^{\varepsilon}$ in a position close to the carbonyl oxygen of the peptidyl-prolyl bond and (ii) $\mathrm{N}^{\varepsilon}$ then acts as a hydrogen-bond donor to stabilize the intermediate of the amide bond. However, this pathway requires the breaking of two hydrogen bonds, a costly process of the reaction. In addition,

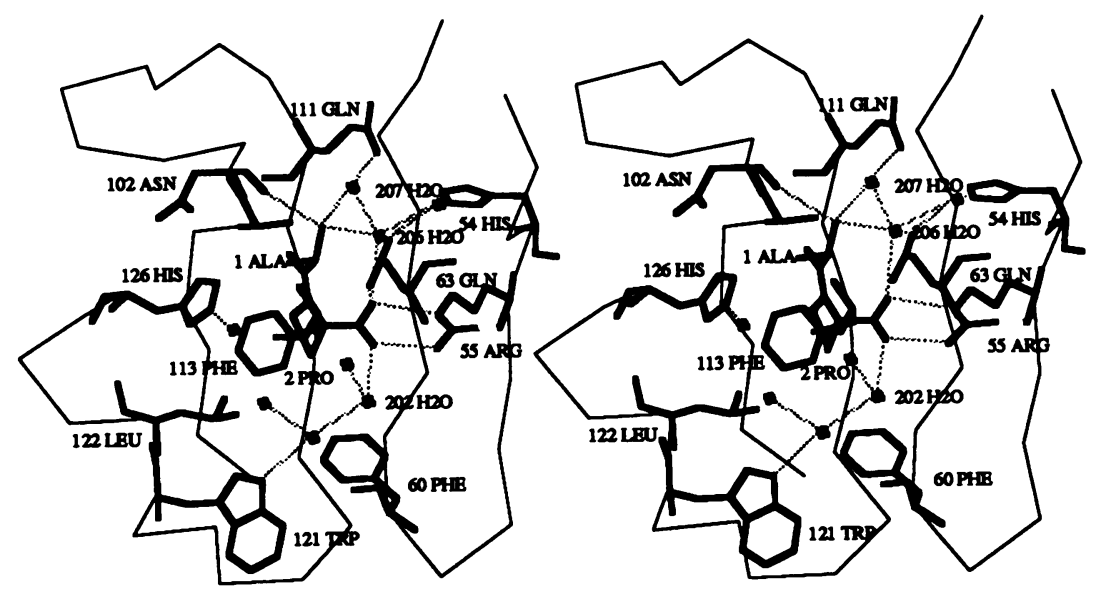

FIG. 3. Stereo drawing of the active site of CyPA with Ala-Pro. Thin lines represent the backbones of Ser ${ }^{51}$ to $\mathrm{Phe}^{67}$ (at right) and Gly to His ${ }^{126}$ (at left), whereas thick lines show non-hydrogen atoms of the active-site residues. The eight water molecules are marked as " $\bullet$," and dotted lines represent hydrogen bonds among substrate, waters, and protein residues. The side chain of proline of the substrate is located in the hydrophobic environment made up of side chains of Phe ${ }^{60}, \mathrm{Met}^{61}, \mathrm{Phe}^{113}$, and Leu ${ }^{122}$, while the $\mathrm{N}$ and C termini of Ala-Pro form hydrogen bonds with the backbone oxygen of $\mathrm{Asn}^{102}$ and the side chains of $\mathrm{Arg}^{55}$ and $\mathrm{Gln}^{63}$ (Table 1). 
the flip of the imidazole plane brings $\mathrm{C}^{\delta}$ of $\mathrm{His}^{126}$ too close to the carbonyl oxygen of $\operatorname{Met}^{100}(2.8 \AA)$. A movement, which is necessary to make a reasonable contact between the imidazole of $\mathrm{His}^{126}$ and the carbonyl oxygen of $\mathrm{Met}^{100}$, will subsequently take $\mathrm{His}^{126}$ away from a position in which it can hydrogen bond to the intermediates of the substrate. Therefore, we do not consider that this mechanism, in which His ${ }^{126}$ stabilizes an intermediate of the cis-trans isomerization, is likely to occur.

Four hydrophobic interactions between $\mathrm{His}^{126}$ and the substrate Ala-Pro in the CyPA/Ala-Pro complex (Table 1) imply that contribution of $\mathrm{His}^{126}$ to the formation of the hydrophobic pocket is essential for the binding of substrate. This assumption would explain the result in which the His ${ }^{126}$ $\rightarrow$ Gln mutation reduced the cis-trans isomerase activity of CyPA to $<1 \%$ of the wild type (18). Since both histidine and glutamine have a $\mathrm{N}^{\varepsilon}$ atom which can be a proton donor for the formation of a hydrogen bond, the conservative mutation from histidine to glutamine may not substantially disturb the hydrogen-bonding pattern or polarity of the enzyme. Instead, because the hydrophobicity of histidine is greater than that of glutamine (19), the His ${ }^{126} \rightarrow$ Gln mutation may significantly decrease the hydrophobicity of the pocket so as to weaken the binding of substrate. In addition, the disappearance of the hydrogen bond between $\mathrm{N}^{\delta}$ of $\mathrm{His}^{126}$ and the carbonyl oxygen of Met $^{100}$ due to the mutation may make the local environment more open and flexible so as to further weaken the hydrophobic pocket of the active site.

Mechanism of cis-trans Isomerization. On the basis of the structure of the CyPA/Ala-Pro complex, we will first comment on existing mechanisms and then propose a solventassisted mechanism for the cis-trans isomerization catalyzed by CyP.

A covalent tetrahedral intermediate, probably as a hemithioorthoamide, was proposed on the basis of results from the chemical modification of the sulfhydryl group (2) and the kinetic $\beta$-deuterium isotope effects (6). However, the observations that the mutation of cysteines to alanines had no effect on the cis-trans isomerase activity (20) and that cysteine residues were excluded from the active site in the three-dimensional structure (11) ruled out the possible formation of the covalent hemithioorthoamide intermediate. Moreover, the present structure reveals no nucleophilic residues or bound waters near the carbonyl carbon of the amide bond. The only groups close to the carbonyl carbon of the amide bond are the side chains of $\operatorname{His}^{126}(3.8 \AA)$ and $\operatorname{Gln}^{63}$ (about $4 \AA$, Fig. 3). These two residues are unlikely to act directly or indirectly (via a water) as a nucleophile attacking the carbonyl carbon. Therefore, the covalent mechanism involving a tetrahedral carbon intermediate might be ruled out.

The observations of the normal kinetic isotope effect, independence of $k_{\text {cat }} / K_{\mathrm{m}}$ from either $\mathrm{pH}(5.5-9.0)$ or the solvent deuterium isotope effect (7), and site-directed mutagenesis of cysteines (20) lead to a distortion mechanism in which noncovalent interactions between CyP and the substrate stabilize the transition state, which is characterized by partial rotation about the $\mathrm{C}-\mathrm{N}$ amide bond (7). However, the structure of the CyPA/Ala-Pro complex shows that only the cis form of Ala-Pro, a ground state rather than a transition state of the amide bond, binds to the enzyme. It is also interesting that the structure of CyPA complexed with the tetrapeptide substrate AAPA has two molecules of AAPA binding to each active site, one of which has a cis conformation of the amide bond and the other of which is partially disordered (14). In short, the distortion binding mechanism is not proven by the structures of CyPA/Ala-Pro and CyPA/ AAPA, although it needs to be determined whether or not other dipeptides, oligopeptides, or protein substrates can bind at an intermediate state.
A third mechanism assumes that the side chains of serine, threonine, or tyrosine protonate or hydrogen bond to the amide nitrogen to stabilize the tetrahedral nitrogen intermediate, on the basis of molecular orbital calculations in which protonation of the amide nitrogen dramatically lowers the barrier for cis-trans isomerization (8). First, this mechanism assumes in principle a general acid/base catalysis and therefore does not appear to be consistent with the kinetic observation that the cis-trans isomerase activity of CyP is independent of pH from 5.5 to 9.0 (7). Second, our CyPA/Ala-Pro structure showed no strong proton-donor groups such as serine, threonine, tyrosine, or bound water close to the amide nitrogen. The only group close to the nitrogen of the peptidylprolyl bond is the amine of $\operatorname{Gn}^{63}$, which is $3.8 \AA$ from the nitrogen. Since a distance of $3.8 \AA$ is too far to form a strong interaction, we do not think that the amine group of $\operatorname{Gln}^{63}$ will donate a proton to the nitrogen of the peptidyl-prolyl bond to form a covalent tetrahedral nitrogen intermediate. Nevertheless, $\operatorname{Gln}^{63}$ may contribute somewhat to the destabilization of the planar conformation of the amide bond if thermal movement or thermodynamic fluctuation brings the amide bond of the substrate and the amine group of $\operatorname{Gln}^{63}$ closer to one another.

The mechanism of catalysis by desolvation was proposed on the basis that transition states of the peptidyl-prolyl bond would be less polar than those of the ground states of substrate and product $(9,10)$. This mechanism is supported by the facts that the rate of cis-trans isomerization was significantly accelerated in nonpolar solvents $(9,10,21)$ and that the hydrophobic pocket is an essential character of the active sites in both structures of CyP and another family of cis-trans isomerase, FK506-binding proteins (22). On the other hand, the structure of the CyPA/Ala-Pro complex also reveals that hydrophilic interactions or hydrogen bonds are critical for both substrate binding and cis-trans isomerase activity. For example, $\mathrm{Arg}^{55}$ forms two hydrogen bonds with the C-terminal oxygens of Ala-Pro (Fig. 3 and Table 1), and the $\mathrm{Arg}^{55} \rightarrow$ Ala mutation maintains $<1 \%$ of the isomerase activity of the wild-type enzyme (18). Therefore, the desolvation of the amide bond or the substrate by binding to the enzyme may be the first step in the cis-trans isomerization. Further actions such as hydrophilic interactions between the enzyme and substrate are required to complete the cis-trans isomerization.

The above hypotheses focus only on the isomerization of the peptidyl-prolyl bond. Since CyP has a broad substrate specificity from dipeptides to long-chain fragments of proteins, a solvent-assisted mechanism, which considers both the rotation of the peptidyl-prolyl bond and the impact of the $\mathbf{N}$ or $\mathbf{C}$ terminus of substrate on the rotation, was proposed on the basis of the unligated structure of CyPA (11). This mechanism envisions the cis-trans isomerization as two actions: $(i)$ rotation of the $\mathrm{C}-\mathrm{N}$ amide bond and (ii) release of $\mathrm{N}$ - or C-terminal residues of substrate from the enzyme. These two actions may be sequential or concerted. The rotation of the amide bond may be carried out while one terminus, either the $\mathbf{N}$ or the $\mathrm{C}$ terminus, is fixed by tight binding to the enzyme. We provide as follows the detailed picture for the first action "rotation of the peptidyl-prolyl bond."

In the structure of CyPA complexed with Ala-Pro, the carbonyl oxygen of the amide bond of the substrate has a strong polar interaction with the backbone nitrogen of Asn ${ }^{102}$ and several van der Waals contacts to $\mathrm{Ala}^{101}$ and His ${ }^{126}$ (Table 1). Since there are no electron-rich residues or groups near the carbonyl oxygen to depolarize the amide bond so as to destabilize the ground state of the substrate, we searched by manually rotating the amide bond for candidates which could stabilize the intermediate state of the amide bond. Indeed, a water molecule, Wat ${ }^{206}$, which binds to the side- 
a

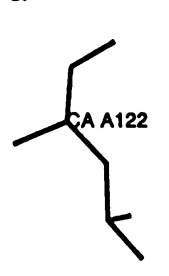

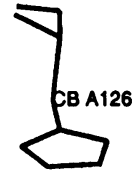
$>$<smiles>CC1(c2ccccc2)CC1</smiles>

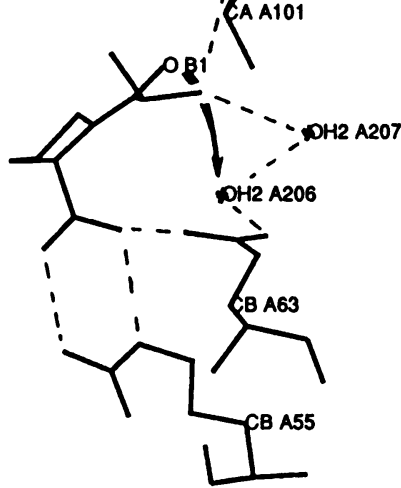

b

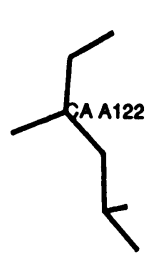

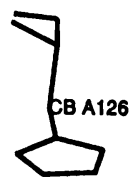
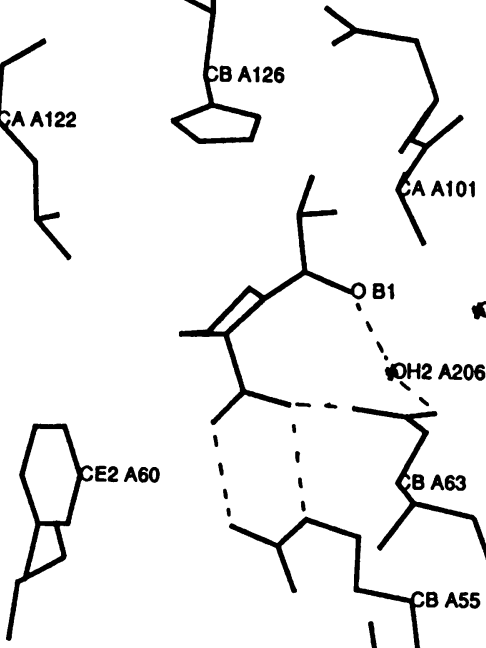

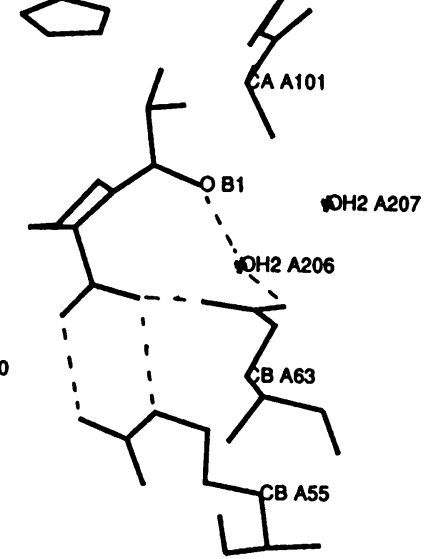

Fig. 4. Simulation of cis-trans isomerization of CyPA. Phe ${ }^{60}, \mathrm{Met}^{61}$ (not shown), Phe ${ }^{113}$ (not shown), Leu ${ }^{122}$, and His ${ }^{126}$ form the hydrophobic pocket of the active site. Some of the hydrogen bonds are marked by broken lines. (a) The ground state of Ala-Pro was observed in the structure of CyPA/Ala-Pro. Arrow represents a potential rotation of the amide bond. (b) A possible intermediate state of Ala-Pro is stabilized by a hydrogen bond between $\mathrm{Wat}^{206}$ and the carbonyl oxygen of the amide bond. The cis-trans isomerization was simulated by rotating the amide bond while the proline residue of Ala-Pro was fixed.

chain oxygen of $\mathrm{Gln}^{63}$ and the N-terminal nitrogen of the substrate, may hydrogen bond to the carbonyl oxygen of the substrate if the peptidyl-prolyl bond is rotated to an angle between $-50^{\circ}$ and $-90^{\circ}$. Since $\mathrm{Wat}^{206}$ is conserved in the unligated structure of CyPA (11) and appears to be a candidate to stabilize the transition state of the amide bond, we propose a solvent-assisted mechanism for the cis-trans isomerization (Fig. 4), in which the peptidyl-prolyl bond is first desolvated in the ground state by binding to the hydrophobic pocket of the active site and later resolvated in the transition state by a bound water molecule.

Simulation of the cis-trans isomerization on the ESV10 graphics system showed that a rotation of the amide bond within $\pm 30^{\circ}$ did not create new contacts between the substrate and the enzyme but substantially brought the carbonyl oxygen of the peptidyl-prolyl bond close to Wat ${ }^{206}$ if the conformation of the protein was kept unchanged. When the peptidyl-prolyl bond was rotated from $-30^{\circ}$ to $-50^{\circ}$, the interaction between the carbonyl oxygen of the substrate and Wat ${ }^{206}$ changed from a van der Waals to a hydrogen bond while the $\mathrm{N}$-terminal nitrogen of the substrate maintained a hydrogen bond to the backbone oxygen of $\mathrm{Ala}^{102}$. However, the distance between $C^{\beta}$ of alanine of the substrate and $C^{\varepsilon}$ of His ${ }^{126}$ dropped from 3.3 to $2.7 \AA$. At $-90^{\circ}$ rotation of the peptidyl-prolyl bond, the distances were $2.5 \AA$ between Wat ${ }^{206}$ and the carbonyl oxygen of Ala-Pro, $3.2 \AA$ between Wat ${ }^{207}$ and the carbonyl oxygen of Ala-Pro, $2.2 \AA$ between the $\mathrm{N}$-terminal nitrogen of the substrate and the carbonyl oxygen of $\mathrm{Ala}^{102}$, and $2.0 \AA$ between $\mathrm{C}^{\beta}$ of alanine of the substrate and $\mathrm{C}^{\varepsilon}$ of His ${ }^{126}$. It is obvious that some energydisfavored contacts arise due to the formation of the substrate intermediates. These disfavored interactions may be relieved by the C-terminal movement subsequent to the rotation of the peptidyl-prolyl bond.

In summary, we rationalize the following pathway for the cis-trans isomerization: (i) the amide bond of Ala-Pro is simultaneously oscillated within $\pm 30^{\circ}$ by thermodynamic fluctuation or thermal movement; (ii) the oscillation consequently brings the carbonyl oxygen of the amide bond close to Wat ${ }^{206}$; (iii) Wat ${ }^{206}$ then induces further transition of the amide bond and stabilizes the intermediate by forming a hydrogen bond with the carbonyl oxygen of the amide bond.

We thank Drs. Christopher T. Walsh and Felicia A. Etzkorn for their help with the CyPA purification, which was performed in Dr. Walsh's laboratory at Harvard Medical School. Drs. Richard V. Wolfenden and Anna Radzicka are acknowledged for their input on the mechanism of the cis-trans isomerization and for their valuable critiques of the manuscript. This work is partially supported by Miles, Inc., at West Haven, CT.

1. Handschumacher, R. E., Harding, M. W., Rice, J. \& Drugge, R. J. (1984) Science 226, 544-547.

2. Fischer, G., Wittmann-Liebold, B., Lang, K., Kiefhaber, T. \& Schmid, F. X. (1989) Nature (London) 337, 476-478.

3. Takahashi, N., Hayano, T. \& Suzuki, M. (1989) Nature (London) 337, 473-475.

4. Schreiber, S. L. (1991) Science 251, 283-287.

5. Fischer, G. \& Schmid, F. X. (1990) Biochemistry 29, 2205-2212.

6. Fischer, G., Berger, E. \& Bang, H. (1989) FEBS Lett. 250, 267-270.

7. Harrison, R. K. \& Stein, R. L. (1990) Biochemistry 29, 1684-1689.

8. Kofron, J. L., Kuzmic, P., Kichore, V., Colon-Bonilla, E. \& Rich, D. H. (1991) Biochemistry 30, 6127-6134

9. Wolfenden, R. \& Radzicka, A. (1991) Chemtracts Biochem. Mol. Biol. 2, 52-54.

10. Radzicka, A., Acheson, S. A. \& Wolfenden, R. (1992) Bioorg. Chem. 20, 382-386.

11. Ke, H. (1992) J. Mol. Biol. 228, 539-550.

12. Ke, H., Zydowsky, L. D., Liu, J. \& Walsh, C. T. (1991) Proc. Natl. Acad. Sci. USA 88, 9483-9487.

13. Kallen, J., Spitzfaden, C., Zurini, M. G. M., Wider, G., Widmer, H., Wüthrich, K. \& Walkinshaw, M. D. (1991) Nature (London) $353,276-279$.

14. Kallen, J. \& Walkinshaw, M. D. (1992) FEBS Lett. 300, 286-290.

15. Jones, T. A. (1982) in Computational Crystallography, ed. Sayer, D. (Oxford, London), pp. 303-317.

16. Brünger, A. T., Kuriyan, J. \& Karplus, M. (1987) Science 235, 458-460.

17. Grathwohl, C. \& Wüthrich, K. (1981) Biopolymers 20, 2623-2633.

18. Zydowsky, L. D., Chang, H. Y., Ferguson, S. B., Ho, S. I., Etzkorn, F. A. \& Walsh, C. T. (1992) Protein Sci. 1, 1092-1099.

19. Radzicka, A. \& Wolfenden, R. (1988) Biochemistry 27, 1664-1670.

20. Liu, J., Albers, M. W., Chen, C. M., Schreiber, S. L. \& Walsh, C. T. (1990) Proc. Natl. Acad. Sci. USA 87, 2304-2308.

21. Eberhardt, E. S., Loh, S. N., Hinck, A. P. \& Raines, R. T. (1992) J. Am. Chem. Soc. 114, 5437-5439.

22. Van Duyne, G. D., Standaert, R. F., Karplus, M., Schreiber, S. L. \& Clardy, J. (1991) Science 252, 839-842. 